\title{
Neuroimaging evidence for cannabinoid modulation of cognition and affect in man
}

\section{Sagnik Bhattacharyya* and Kyra-Verena Sendt}

Department of Psychosis Studies, Institute of Psychiatry, King's College London, London, UK

*Correspondence: sagnik.2.bhattacharyya@kcl.ac.uk

Cannabinoid receptors (CB1 and $\mathrm{CB} 2)$ are ubiquitous within the brain (Wilson and Nicoll, 2002; Eggan and Lewis, 2007). Their distribution and role in the modulation of different neurotransmitter systems (Pertwee and Ross, 2002; Pertwee, 2008a) clearly indicate that cannabinoids are involved in the modulation of different cognitive and emotional processes (Solowij and Michie, 2007). The role of the endocannabinoid system in this has attracted the attention of basic scientists for decades (Zanettini et al., 2011). The modulation of cognitive and emotional processes in man by the extracts of Cannabis sativa ( $C$ sativa), the most commonly used illicit drug consumed by an estimated $4 \%$ of the adult population worldwide (Copeland and Swift, 2009), has also been known for a long time and extensively investigated in experimental and observational studies (Solowij, 1998; Ranganathan and D'Souza, 2006; Solowij and Michie, 2007; Crippa et al., 2009; D'Souza et al., 2009). However, only over the last 20 years has it been possible to precisely investigate the neural basis of the acute effects of cannabinoids on cognition by employing sophisticated neuroimaging techniques (Bhattacharyya et al., 2009a, 2012a; MartinSantos et al., 2010). A renewed interest in the link between regular cannabis use and development of psychotic disorders has provided further impetus, coupled with interest in the therapeutic potential of certain cannabinoids.

Pharmacological challenge studies involving the administration of cannabinoids present in the extract of $C$ sativa or their synthetic counterparts in combination with neuroimaging have served to complement current understanding regarding the role of the endocannabinoid system in regulating human cognitive and emotional processes (Zanettini et al., 2011), to model aspects of various psychiatric illnesses in man and understand their neural underpinnings (Bhattacharyya et al., 2009a). Among the more than 60 different cannabinoids (Mechoulam and Gaoni, 1967) present in the extract of C sativa, delta-9-tetrahydrocanab- inol (THC) is thought to be responsible for most of the psychotropic effects of cannabis (Mechoulam et al., 1970) and modulation of cognitive domains such as learning and memory (Hall and Solowij, 1998; Curran et al., 2002; Ranganathan and D'Souza, 2006), psychomotor control (Hart et al., 2001; McDonald et al., 2003; Ramaekers et al., 2006, 2009), and attention (Hall and Solowij, 1998; Ilan et al., 2004), as evident from systematic acute experimental studies. The purpose of this article is to provide a brief critical overview of current neuroimaging evidence of the acute effects of THC in man as evident from neuroimaging studies. The studies are organized into groups based on neuroimaging domains examined.

\section{MEMORY AND VERBAL LEARNING}

To date, three functional magnetic resonance imaging (fMRI) studies have examined the neural correlates of the effects of cannabinoids on memory processing, the only cognitive domain robustly affected in chronic cannabis users and following acute administration (Grant et al., 2003; Ranganathan and D'Souza, 2006; Solowij and Michie, 2007; D'Souza et al., 2008). Bhattacharyya et al. (2009b) examined the effects of THC on neural activation while healthy occasional cannabis users performed a learning task that involved the repeated presentation of verbal stimuli. Consistent with previous reports (Zeineh et al., 2003), most of the learning under the placebo condition occurred during the first presentation of the encoding block and there was a linear decrement in the engagement of the parahippocampal gyrus, which is involved in the encoding of contextual information about stimuli that may be reactivated later to aid in recollection (Eichenbaum et al., 2007). Administration of THC disrupted the normal linear decrement present with placebo in the engagement of the parahippocampal cortex, which is involved in the encoding of contextual information about stimuli (Eichenbaum et al., 2007). Furthermore, the relationship between the change in parahippocampal activation and memory performance present with placebo was disrupted by THC, consistent with evidence that THC impairs medial temporal function in animals (Robbe et al., 2006; Puighermanal et al., 2009; Wise et al., 2009) and memory performance in animals and man (Curran et al., 2002; D'Souza et al., 2004; Robbe et al., 2006; Puighermanal et al., 2009; Wise et al., 2009). These results may reflect increased demands on encoding under the influence of THC as a result of an impairment in the efficient encoding of contextual information in the medial temporal cortex, which has a central role in relational memory binding (Hannula and Ranganath, 2008). Its activation has been shown previously to correlate with the quantity of novel and successful mnemonic processing (Stern et al., 1996; Brewer et al., 1998; Wagner et al., 1998; Eldridge et al., 2000; Zeineh et al., 2000, 2003). During the recall condition of the task, THC augmented activation in the left medial prefrontal and dorsal anterior cingulate cortex (ACC), areas that have been related to retrieval monitoring and verification (Simons et al., 2005; Fleck et al., 2006). THC also attenuated left rostral ACC and bilateral striatal activation, and its effect in the ventral striatum was directly correlated with the severity of psychotic symptoms induced by it concurrently, demonstrating that the acute induction of psychotic symptoms by THC is related to its effects on striatal function. This study also provided the first human evidence that impairments in learning and memory induced by cannabis are mediated through its effects on medial temporal and prefrontal function.

Subsequently, Bossong et al. (2011) reported an attenuation of activity under the influence of THC in the insula and inferior frontal gyrus on the right side and in the middle occipital gyrus on the left side during the encoding condition of an associative memory task involving pictorial stimuli. During the recall condition, THC enhanced the engagement of the cuneus and 
precuneus. As the authors did not observe any significant effect of THC on task performance, the neural effects may be interpreted as being related to the pharmacological effects of the drug rather than being confounded by differential task performance.

More recently, Bhattacharyya et al. (2012b) employed their previously established design (Bhattacharyya et al., 2009b) and examined the genetic moderation of the neural effects of orally administered THC during memory processing. Variations in genes modulating central dopaminergic neurotransmission, such as AKT1 (rs1130233) and dopamine transporter (DAT1) (40 basepair variable number of tandem repeats in the $3^{\prime}$ untranslated region) were found to modulate the effects of THC on medial temporal, striatal, and midbrain function during encoding and recall conditions. Furthermore, the effects of THC on striatal and midbrain activation during the encoding and recall conditions, respectively, of the verbal memory task were greater in those individuals carrying the risk variants of both the genes compared to the rest.

\section{ATTENTION AND RESPONSE INHIBITION}

O'Leary et al. (2002) examined the neural correlates of the attentional deficits reported following both acute administration and chronic use of cannabis (Hall and Solowij, 1998; Solowij and Michie, 2007). During a dichotic listening task performed by a group of regular abstinent cannabis users they observed an increase in regional cerebral blood flow $(\mathrm{rCBF})$ in the temporal poles bilaterally, cerebellum, insula, and putamen on the right side and the left ventral frontal cortex and a decrease in rCBF in the left superior temporal gyrus, right occipital lobe and bilateral frontal cortical regions areas that form an integral component of the attentional network (Berger and Posner, 2000). In a subsequent study (O'Leary et al., 2007), the authors employed an improved design that allowed them to minimize the carryover effects of THC and reported a significant increase in rCBF bilaterally in the anterior insula, anterior cingulate, orbital frontal lobe, temporal poles, and cerebellum and decrease in rCBF in the mesial occipital lobes and precuneus under the influence of THC.

Borgwardt et al. (2008) examined the neural substrates for the impairments in psychomotor control reported in cannabis users
(Ramaekers et al., 2009; Crean et al., 2011) and reported that administration of THC resulted in a decrease in the normal activation associated with response inhibition in the right inferior frontal gyrus as well as the ACC - key regions implicated in inhibitory control (Garavan et al., 1999; Rubia et al., 2001).

\section{EMOTIONAL AND SENSORY PROCESSING}

Several studies have employed neuroimaging to study the effects of THC on emotional and sensory processing. Phan et al. (2008) investigated the effect of a small dose of THC during the processing of social signals of threat by using angry and fearful faces and reported an attenuation of amygdalar activation. Although this was not associated with any changes in anxiety ratings, the authors interpreted their results as indicative of a potential anxiolytic role of THC. It is likely that lack of a significant anxiogenic effect in this study was related to the lower dose of THC employed by Phan and colleagues as in a subsequent study, Fusar-Poli et al. (2009) reported a significant increase in anxiety ratings under the influence of a higher dose of THC. However, these effects were not associated with modulation of amygdala activity under the influence of THC. Instead, THC produced an increase in engagement of the right inferior parietal lobule and attenuation of engagement of the left medial frontal gyrus while viewing mildly fearful faces. While viewing intensely fearful faces, there was an increase in engagement of the left precuneus and primary sensorimotor corex bilaterally and decrease in engagement of the middle frontal gyrus bilaterally and in the posterior cingulate gyrus. In a subsequent three-way comparison between the effects of THC and cannabdiol, a non-psychoactive ingredient in cannabis, relative to the placebo condition, the same group reported a modulatory effect of THC on amygdalar processing (Bhattacharyya et al., 2010), which was directly correlated with the increase in anxiety induced by it suggesting that the lack of effect on amygdala activation in the former study (Fusar-Poli et al., 2009) may have been related to a modestly powered sample.

Winton-Brown et al. (2011) examined the modulation of activation during auditory and visual processing in healthy subjects as the acute abnormalities in sensory processing (Tart, 1970) under the influence of cannabis are similar to those experienced during psychotic episodes (Koethe et al., 2006). During an auditory processing condition, THC attenuated activation bilaterally in the anterior and posterior superior temporal gyrus and middle temporal gyrus, the insulae and in the supramarginal gyri and in the right inferior frontal gyrus and left cerebellum relative to the placebo condition. During a visual processing condition, THC attenuated activation in the extrastriate visual cortex and enhanced activation in lingual and middle occipital gyri (corresponding to the primary visual cortex) on the right side and parts of the lingual and fusiform gyri extending anteriorly on the left side.

\section{REWARD AND SALIENCE PROCESSING}

Bhattacharyya et al. (2012c) examined the effect of THC on attentional salience processing and its relationship with psychotic symptoms induced under its influence. Employing a visual oddball detection task, they observed that relative to placebo THC attenuated activation in the right caudate but augmented it in the right prefrontal cortex, including the inferior frontal gyrus, during the processing of "salient" oddball stimuli relative to "non-salient" standard stimuli. This was associated with a reduction in response latency to standard relative to oddball stimuli under THC, suggesting that the non-salient standard stimuli may have appeared relatively more salient under the influence of THC. This is consistent with evidence that insignificant sensory stimuli or commonplace conversations acquire new meanings and significance under the influence of cannabis (Tart, 1970). The effect of THC in the right caudate was negatively correlated with the severity of the psychotic symptoms it induced and its effect on response latency. These results provide experimental support for the salience model of psychosis (Kapur, 2003), are consistent with evidence of abnormal salience attribution in patients with schizophrenia (Jensen et al., 2008; Murray et al., 2008) as well as linking aberrant salience attribution and the presence of psychotic symptoms (Roiser et al., 2009). Furthermore, they provide the first evidence that the effects of cannabis on psychosis may be mediated by influencing the neural substrate of attentional salience processing.

van Hell et al. (2012) employed a monetary reward task involving reward anticipation and feedback conditions to 
explore the role of the endocannabinoid system during human reward processing (Gardner, 2005; Solinas et al., 2007). Under the influence of THC there was reduced feedback-related activity in the left inferior parietal cortex and the inferior temporal gyrus bilaterally during the rewarding trials, but no effect during the non-rewarding trials. These neural effects were associated with a trend-level slowing down of the speed of task performance for both the rewarding and neutral trials under THC influence, although this effect was more prominent for the reward trials. This was in contrast to the faster responding to the rewarding relative to the neutral trials observed across all conditions. THC did not have any significant effect on neural activation during the anticipation of reward. This may suggest that under THC influence, salient and rewarding trials may appear less striking (van Hell et al., 2012). This is indicated by a greater slowing down during the rewarding trials as well as attenuation of activation under the influence of THC in the inferior parietal cortex, which functions as a "behavioral integrator" providing a "salience representation" of the external world and signals attentional priority for behaviorally salient signals (Gottlieb, 2007).

\section{CONCLUSION}

Neuroimaging studies reviewed here suggest that, consistent with the polymorphic and heterogeneous nature of the cognitive and symptomatic effects of cannabis, THC has modulatory effects over widely distributed neural networks in man. While the earliest neuroimaging studies involving cannabinoids [reviewed in Bhattacharyya et al. (2009a) and Martin-Santos et al. (2010)] mainly investigated the effects of chronic use or acute administration of cannabis on rCBF, more recent studies have employed neuroimaging technologies with better spatial resolution to investigate the modulation of the neural correlates of cognitive and emotional processes by cannabinoids (Bhattacharyya et al., 2012). These studies demonstrate the neural basis of the effects of THC across a number of cognitive (learning, attention, response inhibition, salience, and reward) and emotional (anxiety) processes (Borgwardt et al., 2008; Bhattacharyya et al., 2009b, 2012a,b). These effects are consistent with the ubiquitous distribution of the main cannabinoid receptor (CB1; Wilson and Nicoll, 2002; Eggan and Lewis, 2007) and are likely to be mediated through the modulation of different neurotransmitter systems (Pertwee, 2008a,b). Delineation of the precise neural mechanisms underlying the distinct and often opposite acute cognitive and symptomatic effects of different cannabinoids in man complements existing evidence from basic science regarding the role of endocannabinoids in cognitive and emotional processing. This may not only help in modeling different aspects of the psychopathology of mental disorders such as schizophrenia and offer insights into their underlying mechanisms, but may suggest potentially new therapeutic targets for drug discovery.

\section{ACKNOWLEDGMENTS}

Sagnik Bhattacharyya has received support from the Medical Research Council, UK (Joint MRC/Priory Clinical research training fellowship; G0501775) and is currently supported by a NIHR Clinician Scientist Award (NIHR CS-11-001).

\section{REFERENCES}

Berger, A., and Posner, M. I. (2000). Pathologies of brain attentional networks. Neurosci. Biobehav. Rev. 24, 3-5.

Bhattacharyya, S., Atakan, Z., Martin-Santos, R., Crippa, J. A., Fusar-Poli, P., and McGuire, P. (2012a). Neural mechanisms for the cannabinoid modulation of cognition and affect in man: a critical review of neuroimaging studies. Curr. Pharm. Des. (in press).

Bhattacharyya, S., Atakan, Z., Martin-Santos, R., Crippa J.A., Kambeitz, J., Prata, D., Willams, S., Brammer, M., Collier, D. A., and McGuire, P. K. (2012b). Preliminary report of biological basis of sensitivity to the effects of cannabis on psychosis: Aktl and DAT1 genotype modulates the effects of delta-9-tetrahydrocannabinol on midbrain and striatal function. Mol. Psychiatry doi: 10.1038/mp.2011.187. [Epub ahead of print].

Bhattacharyya, S., Crippa, J. A., Allen, P., Martin-Santos, R., Borgwardt, S., Fusar-Poli, P., Rubia, K., Kambeitz, J., O'Carroll, C., Seal, M., Giampietro, V., Brammer, M., Zuardi,A. W., Atakan, Z., and McGuire, P. K. (2012c). Induction of psychosis by delta-9-tetrahydrocannbinol reflects modulation of prefrontal and striatal function during attentional salience processing. Arch Gen. Psychiatry 69, 27-36.

Bhattacharyya, S., Crippa, J. A., Martin-Santos, R., Winton-Brown, T., and Fusar-Poli, P. (2009a). Imaging the neural effects of cannabinoids: current status and future opportunities for psychopharmacology. Curr. Pharm. Des. 15, 2603-2614.

Bhattacharyya, S., Fusar-Poli, P., Borgwardt, S., MartinSantos, R., Nosarti, C., O’Carroll, C., Allen, P., Seal, M. L., Fletcher, P. C., Crippa, J. A., Giampietro, V., Mechelli, A., Atakan, Z., and McGuire, P. (2009b). Modulation of mediotemporal and ventrostriatal function in humans by delta-9-tetrahydrocannabi- nol: a neural basis for the effects of cannabis sativa on learning and psychosis. Arch. Gen. Psychiatry 66, 442-451.

Bhattacharyya, S., Morrison, P. D., Fusar-Poli, P., Martin-Santos, R., Borgwardt, S., Winton-Brown, T., Nosarti, C., O'Carroll, C., Seal, M., Allen, P., Mehta, M. A., Stone, J. M., Tunstall, N., Giampietro, V., Kapur, S., Murray, R. M., Zuardi, A. W., Crippa, J. A., Atakan, Z., and McGuire, P. K. (2010). Opposite effects of delta-9-tetrahydrocannabinol and cannabidiol on human brain function and psychopathology. Neuropsychopharmacology 35, 764-774.

Borgwardt, S. J., Allen, P., Bhattacharyya, S., Fusar-Poli, P., Crippa, J. A., Seal, M. L., Fraccaro, V., Atakan, Z., Martin-Santos, R., O'Carroll, C., Rubia, K., and McGuire, P. K. (2008). Neural basis of delta-9-tetrahydrocannabinol and cannabidiol: effects during response inhibition. Biol. Psychiatry 64, 966-973.

Bossong, M. G., Jager, G., van Hell, H. H., Zuurman, L., Jansma, J. M., Mehta, M. A., van Gerven, J. M. A., Kahn, R. S., and Ramsey, N. F. (2011). Effects of 89-tetrahydrocannabinol administration on human encoding and recall memory function: a pharmacological fmri study. J. Cogn. Neurosci. 24, 588-599.

Brewer, J. B., Zhao, Z., Desmond, J. E., Glover, G. H., and Gabrieli, J. D. (1998). Making memories: brain activity that predicts how well visual experience will be remembered. Science 281, 1185-1187.

Copeland, J., and Swift, W. (2009). Cannabis use disorder: epidemiology and management. Int. Rev. Psychiatry 21, 96-103.

Crean, R. D., Crane, N. A., and Mason, B. J. (2011). An evidence based review of acute and long-term effects of cannabis use on executive cognitive functions. $J$. Addict. Med. 5, 1-8.

Crippa, J. A., Zuardi, A. W., Martin-Santos, R., Bhattacharyya, S., Atakan, Z., McGuire, P., and Fusar-Poli, P. (2009). Cannabis and anxiety: a critical review of the evidence. Hum. Psychopharmacol. 24, 515-523.

Curran, H. V., Brignell, C., Fletcher, S., Middleton, P., and Henry, J. (2002). Cognitive and subjective dose-response effects of acute oral delta 9-tetrahydrocannabinol (thc) in infrequent cannabis users. Psychopharmacology (Berl.) 164, 61-70.

D’Souza, D. C., Perry, E., MacDougall, L., Ammerman, Y., Cooper, T., Wu, Y. T., Braley, G., Gueorguieva, R., and Krystal, J. H. (2004). The psychotomimetic effects of intravenous delta-9-tetrahydrocannabinol in healthy individuals: implications for psychosis. Neuropsychopharmacology 29, 1558-1572.

D’Souza, D. C., Ranganathan, M., Braley, G., Gueorguieva, R., Zimolo, Z., Cooper, T., Perry, E., and Krystal, J. (2008). Blunted psychotomimetic and amnestic effects of delta-9-tetrahydrocannabinol in frequent users of cannabis. Neuropsychopharmacology 33, 2505-2516.

D’Souza, D.C., Sewell, R. A., and Ranganathan, M. (2009). Cannabis and psychosis/schizophrenia: human studies. Eur. Arch. Psychiatry Clin. Neurosci. 259, 413-431.

Eggan, S.M., and Lewis, D.A.(2007).Immunocytochemical distribution of the cannabinoid cbl receptor in the primate neocortex: a regional and laminar analysis. Cereb. Cortex 17, 175-191.

Eichenbaum, H., Yonelinas, A. P., and Ranganath, C. (2007). The medial temporal lobe and recognition memory. Annu. Rev. Neurosci. 30, 123-152.

Eldridge, L. L., Knowlton, B. J., Furmanski, C. S., Bookheimer,S.Y., and Engel,S.A.(2000). Remembering 
episodes: a selective role for the hippocampus during retrieval. Nat. Neurosci. 3, 1149-1152.

Fleck, M. S., Daselaar, S. M., Dobbins, I. G., and Cabeza, R. (2006). Role of prefrontal and anterior cingulate regions in decision-making processes shared by memory and nonmemory tasks. Cereb. Cortex 16, 1623-1630.

Fusar-Poli, P., Crippa, J.A., Bhattacharyya, S., Borgwardt, S. J., Allen, P., Martin-Santos, R., Seal, M., Surguladze, S. A., O'Carrol, C., Atakan, Z., Zuardi, A. W., and McGuire, P. K. (2009). Distinct effects of \{delta\}9tetrahydrocannabinol and cannabidiol on neural activation during emotional processing. Arch. Gen. Psychiatry 66, 95-105.

Garavan, H., Ross, T. J., and Stein, E. A. (1999). Right hemispheric dominance of inhibitory control: an event-related functional mri study. Proc. Natl. Acad. Sci. U.S.A. 96, 8301-8306.

Gardner, E. L. (2005). Endocannabinoid signaling system and brain reward: emphasis on dopamine. Pharmacol. Biochem. Behav. 81, 263-284.

Gottlieb, J. (2007). From thought to action: the parietal cortex as a bridge between perception, action, and cognition. Neuron 53, 9-16.

Grant, I., Gonzalez, R., Carey, C. L., Natarajan, L., and Wolfson, T. (2003). Non-acute (residual) neurocognitive effects of cannabis use: a meta-analytic study. $J$. Int. Neuropsychol. Soc. 9, 679-689.

Hall, W., and Solowij, N. (1998). Adverse effects of cannabis. Lancet 352, 1611-1616.

Hannula, D. E., and Ranganath, C. (2008). Medial temporal lobe activity predicts successful relational memory binding. J. Neurosci. 28, 116-124.

Hart, C. L., van Gorp, W., Haney, M., Foltin, R. W., and Fischman, M. W. (2001). Effects of acute smoked marijuana on complex cognitive performance. Neuropsychopharmacology 25, 757-765.

Ilan, A. B., Smith, M. E., and Gevins, A. (2004). Effects of marijuana on neurophysiological signals of working and episodic memory. Psychopharmacology (Berl.) 176, 214-222.

Jensen, J., Willeit, M., Zipursky, R. B., Savina, I., Smith, A. J., Menon, M., Crawley, A. P., and Kapur, S. (2008). The formation of abnormal associations in schizophrenia: neural and behavioral evidence. Neuropsychopharmacology 33, 473-479.

Kapur, S. (2003). Psychosis as a state of aberrant salience: a framework linking biology, phenomenology, and pharmacology in schizophrenia. Am. J. Psychiatry 160, 13-23.

Koethe, D., Gerth, C. W., Neatby, M. A., Haensel, A., Thies, M., Schneider, U., Emrich, H. M., Klosterkotter, J., Schultze-Lutter, F., and Leweke, F. M. (2006). Disturbances of visual information processing in early states of psychosis and experimental delta9-tetrahydrocannabinol altered states of consciousness. Schizophr. Res. 88, 142-150.

Martin-Santos, R., Fagundo, A. B., Crippa, J.A.,Atakan,Z., Bhattacharyya, S., Allen, P., Fusar-Poli, P., Borgwardt, S., Seal, M., Busatto, G. F., and McGuire, P. (2010). Neuroimaging in cannabis use: a systematic review of the literature. Psychol. Med. 40, 383-398.

McDonald, J., Schleifer, L., Richards, J. B., and de Wit, H. (2003). Effects of the on behavioral measures of impulsivity in humans. Neuropsychopharmacology 28, 1356-1365.

Mechoulam, R., and Gaoni, Y. (1967). Recent advances in the chemistry of hashish. Fortschr. Chem. Org. Naturst. 25, 175-213.
Mechoulam, R., Shani, A., Edery, H., and Grunfeld, Y (1970). Chemical basis of hashish activity. Science $169,611-612$

Murray, G. K., Corlett, P. R., Clark, L., Pessiglione, M., Blackwell, A. D., Honey, G., Jones, P. B., Bullmore, E. T. Robbins, T. W., and Fletcher, P. C. (2008). Substantia nigra/ventral tegmental reward prediction error disruption in psychosis. Mol. Psychiatry 239, 267-276.

O’Leary, D. S., Block, R. I., Koeppel, J. A., Flaum, M. Schultz, S. K., Andreasen, N. C., Ponto, L. B., Watkins, G. L., Hurtig, R. R., and Hichwa, R. D. (2002). Effects of smoking marijuana on brain perfusion and cognition. Neuropsychopharmacology 26, 802-816.

O’Leary, D. S., Block, R. I., Koeppel, J. A., Schultz, S. K. Magnotta, V. A., Ponto, L. B., Watkins, G. L., and Hichwa, R. D. (2007). Effects of smoking marijuana on focal attention and brain blood flow. Hum. Psychopharmacol. 22, 135-148.

Pertwee, R. G. (2008a). The diverse cb1 and cb2 receptor pharmacology of three plant cannabinoids: delta9tetrahydrocannabinol, cannabidiol and delta9-tetrahydrocannabivarin. Br. J. Pharmacol. 153, 199-215.

Pertwee, R. G. (2008b). Ligands that target cannabinoid receptors in the brain: from thc to anandamide and beyond. Addict. Biol. 13, 147-159.

Pertwee, R. G., and Ross, R.A. (2002). Cannabinoid receptors and their ligands. Prostaglandins Leukot. Essent. Fatty Acids 66, 101-121.

Phan, K. L., Angstadt, M., Golden, J., Onyewuenyi, I., Popovska, A., and de Wit, H. (2008). Cannabinoid modulation of amygdala reactivity to social signals of threat in humans. J. Neurosci. 28, 2313-2319.

Puighermanal, E., Marsicano, G., Busquets-Garcia,A., Lutz, B., Maldonado, R., and Ozaita, A. (2009). Cannabinoid modulation of hippocampallong-term memory is mediated by mtor signaling. Nat. Neurosci. 12, 1152-1158.

Ramaekers, J. G., Kauert, G., Theunissen, E. L., Toennes, S. W., and Moeller, M. R. (2009). Neurocognitive performance during acute THC intoxication in heavy and occasional cannabis users. J. Psychopharmacol. (Oxford) 23, 266-277.

Ramaekers, J. G., Moeller, M. R., van Ruitenbeek, P., Theunissen, E. L., Schneider, E., and Kauert, G. (2006). Cognition and motor control as a function of delta9the concentration in serum and oral fluid: limits of impairment. Drug Alcohol Depend. 85, 114-122.

Ranganathan, M., and D'Souza, D. C. (2006). The acute effects of cannabinoids on memory in humans: a review. Psychopharmacology (Berl.) 188, 425-444.

Robbe, D., Montgomery, S. M., Thome, A., RuedaOrozco, P. E., McNaughton, B. L., and Buzsaki, G. (2006). Cannabinoids reveal importance of spike timing coordination in hippocampal function. Nat. Neurosci. 9, 1526-1533.

Roiser, J. P., Stephan, K. E., den Ouden, H. E., Barnes, T. R., Friston, K. J., and Joyce, E. M. (2009). Do patients with schizophrenia exhibit aberrant salience? Psychol. Med. 39, 199-209.

Rubia, K., Russell, T., Overmeyer, S., Brammer, M. J., Bullmore, E. T., Sharma, T., Simmons, A., Williams, S. C., Giampietro, V., Andrew, C. M., and Taylor, E. (2001). Mapping motor inhibition: conjunctive brain activations across different versions of go/no-go and stop tasks. Neuroimage 13, 250-261.

Simons, J. S., Gilbert, S. J., Owen, A. M., Fletcher, P. C., and Burgess, P. W. (2005). Distinct roles for lateral and medial anterior prefrontal cortex in contextual recollection. J. Neurophysiol. 94, 813-820.
Solinas, M., Yasar, S., and Goldberg, S. R. (2007). Endocannabinoid system involvement in brain reward processes related to drug abuse. Pharmacol. Res. 56, 393-405.

Solowij, N. (1998). Cannabis and Cognitive Functioning. Cambridge: Cambridge University Press.

Solowij, N., and Michie, P. T. (2007). Cannabis and cognitive dysfunction: parallels with endophenotypes of schizophrenia? J. Psychiatry Neurosci. 32, 30-52.

Stern, C. E., Corkin, S., Gonzalez, R. G., Guimaraes, A. R., Baker, J. R., Jennings, P. J., Carr, C. A., Sugiura, R. M., Vedantham, V., and Rosen, B. R. (1996). The hippocampal formation participates in novel picture encoding: evidence from functional magnetic resonance imaging. Proc. Natl. Acad. Sci. U.S.A. 93, 8660-8665.

Tart, C. T. (1970). Marijuana intoxication common experiences. Nature 226, 701-704.

van Hell, H. H., Jager, G., Bossong, M. G., Brouwer, A., Jansma, J. M., Zuurman, L., van Gerven, J., Kahn, R. S., and Ramsey, N. F. (2012). Involvement of the endocannabinoid system in reward processing in the human brain. Psychopharmacology (Berl.) 219 , 981-990.

Wagner, A. D., Schacter, D. L., Rotte, M., Koutstaal, W., Maril, A., Dale, A. M., Rosen, B. R., and Buckner, R. L. (1998). Building memories: remembering and forgetting of verbal experiences as predicted by brain activity. Science 281, 1188-1191.

Wilson, R. I., and Nicoll, R. A. (2002). Endocannabinoid signaling in the brain. Science 296, 678-682.

Winton-Brown, T. T., Allen, P., Bhattacharyya, S., Borgwardt, S. J., Fusar-Poli, P., Crippa, J. A., Seal M. L., Martin-Santos, R., Ffytche, D., Zuardi, A. W., Atakan, Z., and McGuire, P. K. (2011). Modulation of auditory and visual processing by delta-9-tetrahydrocannabinol and cannabidiol: an fmri study. Neuropsychopharmacology 36, 1340-1348.

Wise, L. E., Thorpe, A. J., and Lichtman, A. H. (2009). Hippocampal $\mathrm{cb}(1)$ receptors mediate the memory impairing effects of delta(9)-tetrahydrocannabinol. Neuropsychopharmacology 34, 2072-2080.

Zanettini, C., Panlilio, L. V., Alicki, M., Goldberg, S. R., Haller, J., and Yasar, S. (2011). Effects of endocannabinoid system modulation on cognitive and emotional behavior. Front. Behav. Neurosci. 5:57. doi: 10.3389/ fnbeh.2011.00057

Zeineh, M. M., Engel, S. A., and Bookheimer, S.Y. (2000). Application of cortical unfolding techniques to functional mri of the human hippocampal region. Neuroimage 11, 668-683.

Zeineh, M. M., Engel, S. A., Thompson, P. M., and Bookheimer, S.Y. (2003). Dynamics of the hippocampus during encoding and retrieval of face-name pairs. Science 299, 577-580.

Received: 03 May 2012; accepted: 05 May 2012; published online: 25 May 2012.

Citation: Bhattacharyya $S$ and Sendt K-V (2012) Neuroimaging evidence for cannabinoid modulation of cognition and affect in man. Front. Behav. Neurosci. 6:22. doi: 10.3389/fnbeh.2012.00022

Copyright (c) 2012 Bhattacharyya and Sendt. This is an open-access article distributed under the terms of the Creative Commons Attribution Non Commercial License, which permits non-commercial use, distribution, and reproduction in other forums, provided the original authors and source are credited. 